\title{
Noninvasive Transcranial Clot Lysis Using High Intensity Focused Ultrasound
}

\author{
T Hölscher ${ }^{1,2 *}$, DJ Fisher ${ }^{1}$, R Raman ${ }^{2,3}$, K Ernstrom ${ }^{3}$, E Zadicario ${ }^{4}$, WG Bradley ${ }^{1}$ and A Voie ${ }^{1}$
}

${ }^{1}$ Department of Radiology, University of California, San Diego, USA

${ }^{2}$ Department of Neurosciences, University of California, San Diego, USA

${ }^{3}$ Department of Familyand Preventive Medicine, University of California, San Diego, USA

${ }^{4}$ InSightec, Inc., Tirat Carmel, Israel

\begin{abstract}
Background: Initial data using transcranial ultrasound in combination with tissue Plasminogen Activator (tPA) for sonothrombolysis in stroke are promising. However, absorption and defocusing of the ultrasound beam occur during transskull insonation, limiting the efficiency of this approach to high extent. The purpose of this in vitro study was to investigate whether transcranial sonothrombolysis can be achieved using High Intensity Focused Ultrasound (HIFU) without the use of tPA.
\end{abstract}

Methods and Results: Transskull clot lysis $(\mathrm{N}=420)$ using a novel HIFU head system was performed using an in vitro flow model. Experiments were performed using varying acoustic output powers, changing flow conditions, and different cadaveric skulls. Human whole blood clots (3 hours incubation) could be lysed within less than 30 seconds and in absence of further lytic agents. Thrombolysis efficacy was dependent on transskull signal absorption, acoustic output power and flow conditions and differed widely between a minimum of $6.21 \%$ and a maximum of $77.26 \%$. Transskull insonation with HIFU did not lead to significant focus beam distortion.

Conclusions: The use of transskull HIFU for immediate clot lysis without the need of further drugs and disregarding individual skull bone characteristics is feasible in vitro.

\section{Introduction}

Stroke is the second most common cause of death worldwide. 800,000 U.S. citizens suffer from stroke every year. Tissue Plasminogen Activator (tPA) is the only FDA approved drug for stroke treatment. Due to the restricted time window and various exclusion criteria less than $3 \%$ of all stroke victims are currently receiving tPA therapy. Less than $40 \%$ of stroke patients treated with tPA demonstrate good clinical recovery on the long term. First data showing that the use of transcranial ultrasound (US) in combination with tPA administration improves early recanalization is very promising [1-10]. Reversible disaggregation of fibrin fibers, improved distribution of plasminogen and PA within a blood clot as well as cavitational mechanisms have been described explaining why ultrasound enhances clot lysis [1-5]. However, the patient population which might benefit is still less than $3 \%$ of all stroke victims.

A major limitation of all transcranial US techniques is the acoustic signal absorption and beam distortion, due to the skull bone. Both have been described as limiting factors already in the 1950's and 1960's [1113]. Ultrasound beams are widely and irregularly scattered by the bone and the brain [14]. Innovative, transcranial High Intensity Focused Ultrasound (HIFU) systems, however, might be able to overcome the problems of beam distortion and phase aberration. An ExAblate ${ }^{\mathrm{TM}} 4000$ High Intensity Focused Ultrasound (HIFU) headsystem (InSightec Inc., Tirat Carmel/Israel), equipped with a transcranial transducer, was used for this study. The device is further described in the Methods section.

If thrombolysis could be achieved mechanically without the use of tPA would be favorable with regard to potential drug related side effects and time restrictions. To achieve thrombolysis within seconds rather than hours, as is currently standard in sonothrombolysis trials using diagnostic US [7,10,15-17], might be beneficial with regard to total US exposure time and potential related tissue damage. Further, US energy would be applied at the target site only and within a very small, predefined volume whereas the vast majority of brain tissue would be minimally affected. This is not the case with conventional or customized [18] transcranial US systems currently used for the purpose of sonothrombolysis.

The goal of this in vitro study was to investigate whether noninvasive transcranial sonothrombolysis can be achieved using the ExAblate ${ }^{\mathrm{TM}}$ 4000 HIFU headsystem.

\section{Methods}

\section{Ultrasound system and parameters}

An ExAblate ${ }^{\mathrm{TM}} 4000$ High Intensity Focused Ultrasound (HIFU) headsystem (InSightec Inc., Tirat Carmel/Israel), equipped with a transcranial transducer, was used for this study. The system has been developed for brain applications. Key component of this system is a hemispheric phased array transducer with 1,000 single piezo elements which can be operated independently. Different from conventional US systems the ExAblate $^{\mathrm{TM}} 4000$ does not produce a single beam which has to pass the skull at a certain location (ie temporal bone window) and travel through brain tissue until it hits the target from one direction. The geometry of the HIFU system has been chosen to insonate through

*Corresponding author: Thilo Hoelscher, Associate Professor, Departments of Radiology and Neurosciences, University of California, San Diego, 200 West Arbor Drive, San Diego, CA 92103-8756, Tel: +1-619-543-5251; Fax: +1-619543-6372; E-mail: thoelscher@ucsd.edu

Received May 19, 2011; Accepted July 15, 2011; Published September 20, 2011

Citation: Hölscher T, Fisher DJ, Raman R, Ernstrom K, Zadicario E, et al. (2011) Noninvasive Transcranial Clot Lysis Using High Intensity Focused Ultrasound. J Neurol Neurophysiol S1. doi:10.4172/2155-9562.S1-002

Copyright: (C) 2011 Hölscher T, et al. This is an open-access article distributed under the terms of the Creative Commons Attribution License, which permits unrestricted use, distribution, and reproduction in any medium, provided the original author and source are credited. 
Citation: Hölscher T, Fisher DJ, Raman R, Ernstrom K, Zadicario E, et al. (2011) Noninvasive Transcranial Clot Lysis Using High Intensity Focused Ultrasound. J Neurol Neurophysiol S1. doi:10.4172/2155-9562.S1-002

the entire skull with 1,000 individual beams. Each individual beam has a significantly lower intensity compared to a conventional US beam. However the sum of all beams leads to a high intensity, three dimensional beam focus in the center of the transducer. A sharp focus (radius: $2.0 \mathrm{~mm}$ in $\mathrm{X} / \mathrm{Y}$ - and $3.0 \mathrm{~mm}$ in $\mathrm{Z}$-orientation) can be generated located in the center of the transducer and steered electronically in a radius of $3.0 \mathrm{~cm}$ in any direction without loosing its shape (Figure 1). Due to the three dimensionality of the beam focus, the target (ie thrombus) is insonated from all directions within the hemisphere.

In the clinical setting, MRI guidance is used for neuronavigation purposes and to steer the focus beam onto the target structure. The clinical set up is shown in Figure 2.

\section{Clot preparation}

Human venous whole blood was drawn from healthy, unmedicated donors (IRB protocol is active) and transferred into citrate tubes. $0.5 \mathrm{ml}$ citrate blood was mixed with $40 \mu \mathrm{CaCl}_{2}(210 \mathrm{mmol} / \mathrm{l})$ and transferred into a boro-silicated glass tube, which had a 2-0 silk thread suspended inside. The thrombi had an average weight of $0.2519 \mathrm{~g} \pm 7 \%$. The average length of each thrombus was about $2.5 \mathrm{~cm}$. Thrombi were incubated for 3.0 hours in a pre heated $\left(37^{\circ} \mathrm{C}\right)$ waterbath and then transferred into a PE test tube (Advanced Polymers, Inc., Salem NH). The PE tubing had an inner diameter of $4.3 \mathrm{~mm}$, a wall thickness of $25.4 \mu \mathrm{m}$ and preferred acoustic properties for US testing. The loose end of the silk thread was fixed upstream inside the flow system to keep the thrombus in place. The PE tube was connected to a non circulating flow system, using a peristaltic pump. Deionized water was used as a fluid medium, using a flow rate of $10 \mathrm{ml} / \mathrm{min}$.

\section{Experimental set up}

Cadaveric skull specimens were mounted on an acrylic frame and placed upside down into the degassed water filled cavity of the hemispheric transducer. A 3D step motor system was used to navigate a pointer and to physically localize the focus in the center of the hemispheric transducer. The thrombus inside the test tube was placed

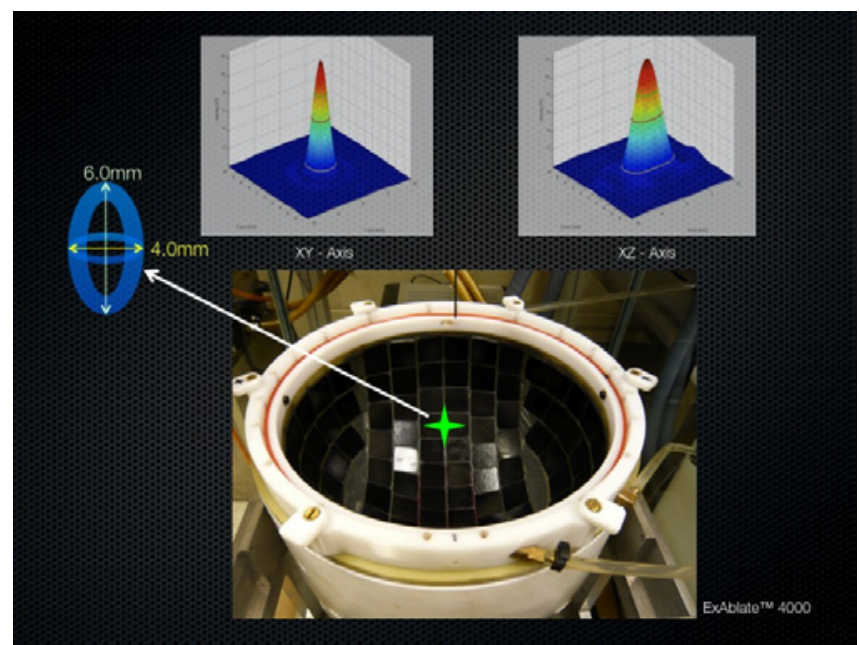

Figure 1: Top view inside the hemispheric transducer of the ExAblate ${ }^{\mathrm{TM}} 4000$ HIFU headsystem. Each black tile contains 9 single ultrasound transmitting elements (total $\mathrm{N}=1,000$ ). All elements transmit towards the center of the transducer (green star), creating a sharp focus beam of $4.0 \mathrm{~mm}$ diameter in lateral and $6.0 \mathrm{~mm}$ in elevational orientation. Parametric images of the focus, based on acoustic measurements, are given in XY (upper left) - and Z (upper right) - orientation

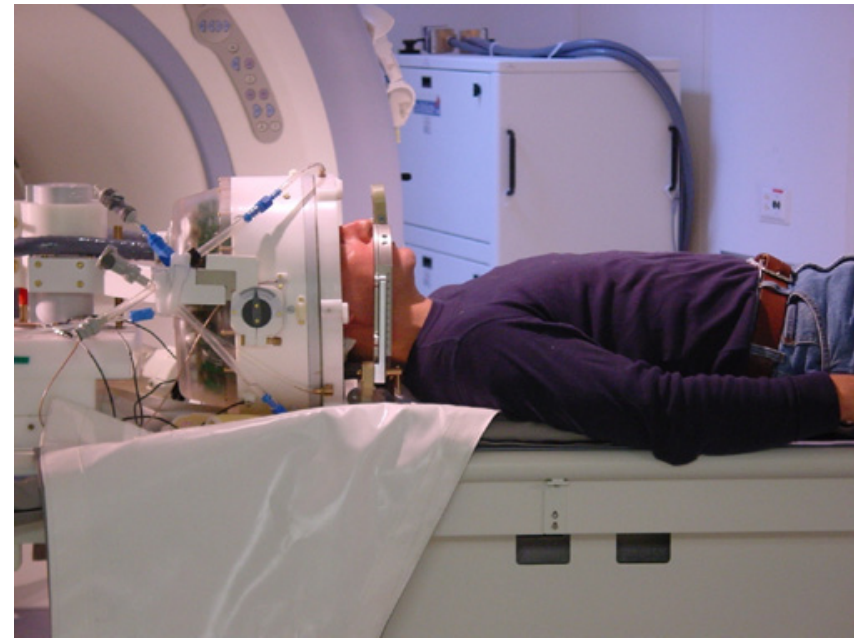

Figure 2: ExAblate ${ }^{\mathrm{TM}} 4000 \mathrm{HIFU}$ headsystem patient set up prior to positioning inside the MRI scanner.

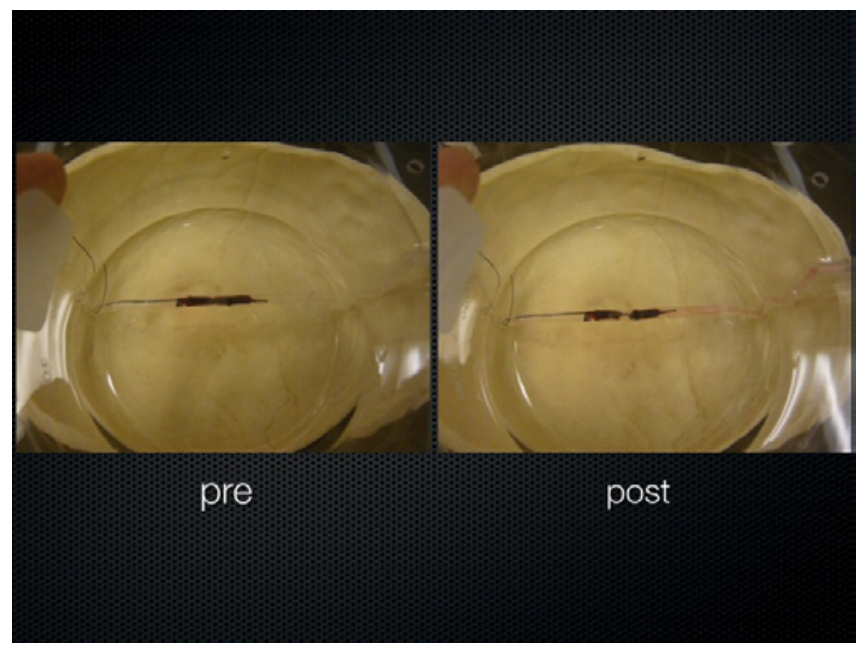

Figure 3: Top view inside the water filled hemispheric HIFU transducer with human cadaveric skull and a thrombus inside the test tube in place. Left - pre HIFU insonation. Right - post HIFU insonation. Please note the center gap in the thrombus on the right.

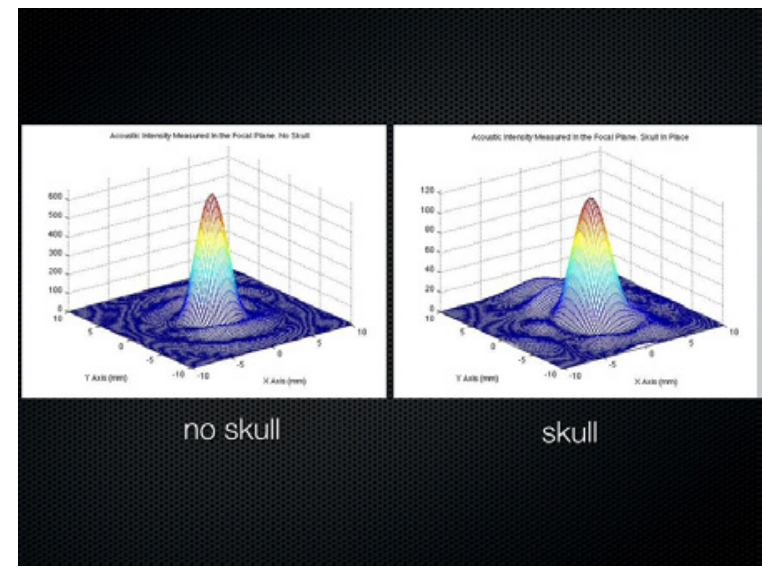

Figure 4: -3 decibel beam areas plotted as parametric images. Left - beam area without skull in place. Right - beam area with skull in place. 

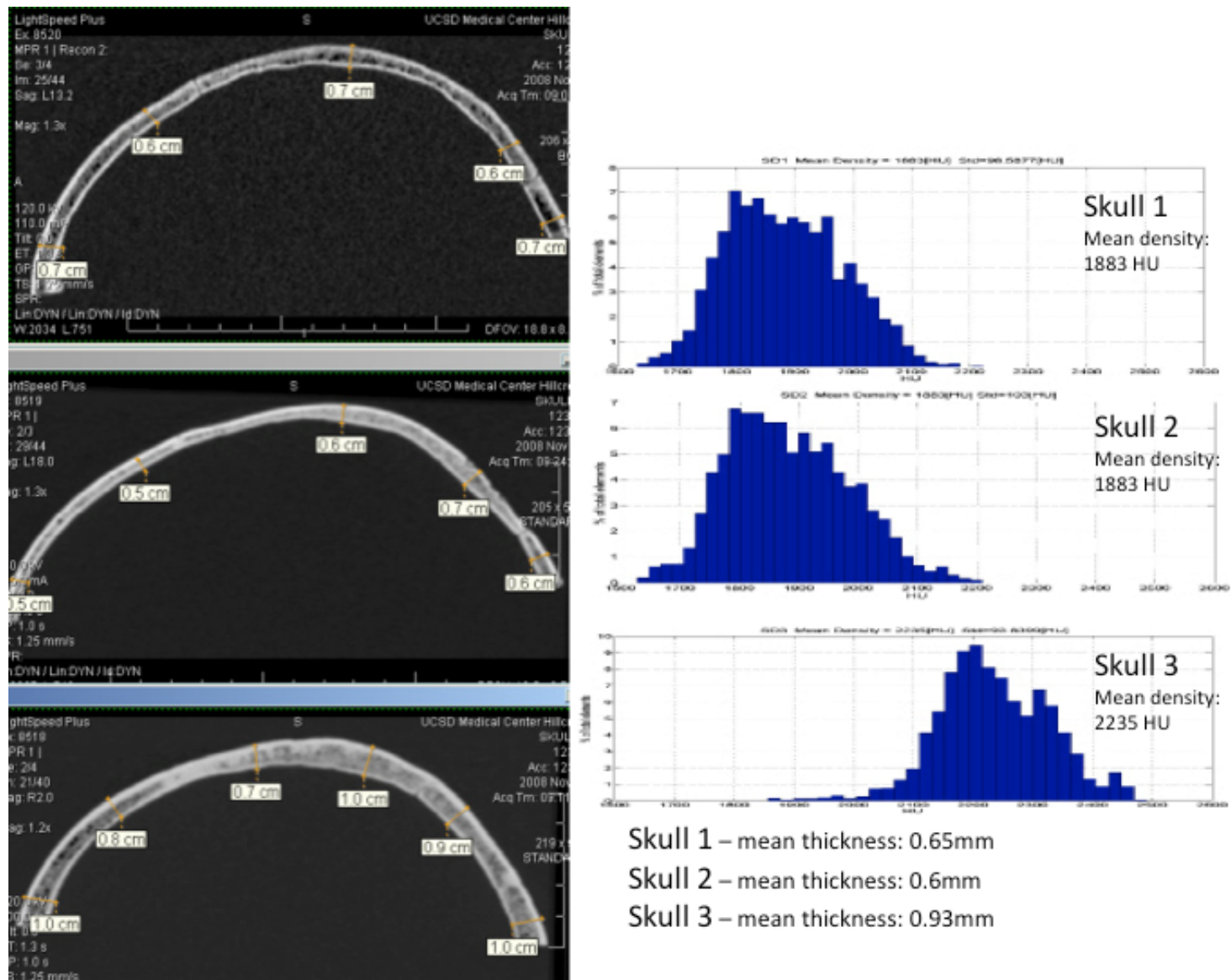

Skull 1 - mean thickness: $0.65 \mathrm{~mm}$

Skull 2 - mean thickness: $0.6 \mathrm{~mm}$

Skull 3 -mean thickness: $0.93 \mathrm{~mm}$

Figure 5: Representative CT images of the 3 human calvaria (Skull \#1-\#3), including bone thickness and radio density graphs for each specimen.

manually at the focus, inside the cavity of the skull. For the experiments the clots were exposed to either circulating, pulsatile flow $(10 \mathrm{ml} / \mathrm{min})$ or no flow $(0 \mathrm{ml} / \mathrm{min})$ to reproduce flow stasis. The latter was mimicked by simply turning off the flow pump.

\section{D computertomography data acquisition}

Prior to the CT data acquisition (Discovery CT750 HD, 64 slice CT scanner, GE Healthcare) the skull calvaria specimens, provided by the UCSD Division of Anatomy, were degassed for 96 hours. Degassing was performed using a custom acrylic vacuum chamber, filled with deionized water and placed under house vacuum $(-12$ inches $\mathrm{Hg})$. After degassing, a 3D data acquisition with a slice thickness and pixel dimension of $0.625 \mathrm{~mm}$ and no spacing was performed. Reconstruction was done using a bone kernel. After CT acquisition the DICOM data were copied to a CD or memory stick to be uploaded onto a dedicated server at the Brain Ultrasound Research Lab.

\section{Acoustic field measurements}

For this project a dedicated sound field scanner was used. AIMS (ONDA Inc., Sunnyvale/California, USA) is a precision scanning system mounted on a tank that allows measuring and mapping of acoustic fields in liquids. It is suitable for medical imaging, pulsed and CW Doppler, therapeutic devices and industrial ultrasound. To acquire the acoustic data a custom-built hydrophone (Y-120S High Intensity Hydrophone, $50 \mathrm{kHz}-1.9 \mathrm{MHz}$ bandwidth, Sonic Concepts, Bothell, WA) was used. At focus, for each skull, the acoustic parameters a) spatial peak temporal average intensity, b) peak rarefractional pressure and c) peak positive pressure as well as the -3 decibel $(\mathrm{dB})$ focus area were assessed. The acoustic data for all 3 calvaria is displayed in Table 1.

\section{Statistical analysis}

To test for pre/post weight loss (in grams) and to test whether different skulls affect clot weight loss pair-wise comparisons were done using Student's t-tests. Bonferroni method was used to adjust for multiple comparison. To test for the effect of varying intensity conditions, a Kruskal-Wallis test was used to compare overall differences in clot weight loss between conditions, followed by pairwise comparisons using a Wilcoxon Rank Sum test with a Holm's adjustment for multiple comparisons.

\section{Results}

Overall, a total of $\mathrm{N}=420$ sonothrombolysis experiments were performed. Since we aimed to answer different research questions we suggest to break down the results presentation according to the following topics:

\section{Does HIFU cause transcranial sonothrombolysis?}

Depending on skull characteristics, acoustic energy and flow mechanics the amount of weight loss after HIFU exposure differed widely between a minimum of $6.21 \%$ and a maximum of $77.26 \%$. However, in all of the $\mathrm{N}=420$ in vitro experiments significant clot lysis could be achieved after 30 seconds of HIFU insonation and without the use of a lytic agent (Figure 3). 


\section{Does defocusing occur during transcranial HIFU?}

Comparing the focus dimensions (ie. $-3 \mathrm{~dB}$ focus area) and the focus location before and after insonation through human cadaveric skulls it could be demonstrated that there is little difference between pre/ post transcranial HIFU exposure (Figure 4). Due to signal absorption caused by the skull bone the signal intensity as well as negative/positive pressure values differ significantly between pre/post scenarios, as expected (Table 1).

\section{Is HIFU sonothrombolysis efficacy depending on skull characteristics?}

To answer this question we performed sonothrombolysis experiments using 3 different human calvaria $(\mathrm{N}=126)$. For comparison, the HIFU operating parameter settings remained the same for the 3 samples:

- Acoustic output power (AP):

$270 \mathrm{~W}$

- Duty cycle (DC):

$50 \%$

- Pulse width (PW):

$200 \mathrm{~ms}$

- HIFU duration (ID):

$30 \mathrm{sec}$

For Skull \#1 $(\mathrm{N}=44)$, the average weight loss was $42.1 \pm 16.2 \%$, compared to $35.6 \pm 14.2 \%$ for Skull \#2 $(\mathrm{N}=41)$ and $10.3 \pm 4.4 \%$ for Skull \#3 $(\mathrm{N}=41)$. For all three series individually, the average weight loss was statistical significant $(\mathrm{p}<0.001)$. Clots treated using Skull \#1 and \#2 lost significantly more weight compared to Skull \#3 clots (both $\mathrm{p}<0.001$ ). A smaller percent weight loss was seen in the Skull \#2 compared to the Skull \#1. This comparison was not found to be statistically significant adjusting for multiple comparisons ( $\mathrm{p}=0.051)$ (Table 2).

Analyzing the CT data we found that Skull \#1 and Skull \#2 were very similar in both bone thickness (mean $5.51 \mathrm{~mm}$ vs. $5.49 \mathrm{~mm}$ ) and

\begin{tabular}{|l|l|l|l|}
\hline & $\begin{array}{l}\text { Intensity } \\
(\text { Watt/cm }\end{array}$ & $\begin{array}{l}\text { Peak Negative Pressure } \\
(\text { Mega Pascal) }\end{array}$ & $\begin{array}{l}\text { Peak Positive Pressure } \\
\text { (Mega Pascal) }\end{array}$ \\
\hline no Skull & 888 & 8.01 & 7.39 \\
\hline Skull \#1 & 111 & 2.81 & 2.75 \\
\hline Skull \#2 & 160 & 3.33 & 3.22 \\
\hline Skull \#3 & 79 & 2.39 & 2.29 \\
\hline
\end{tabular}

*measured at AP: $270 \mathrm{~W}, \mathrm{DC}: 50 \%$, PW: $200 \mathrm{~ms}$

Table 1: Acoustic Measurements at Focus*

\begin{tabular}{|l|l|l|l|l|l|l|l|}
\hline & N & Min. & Median & Mean & Max. & SD & P value \\
\hline Skull \#1 & 44 & 14.50 & 40.04 & 42.12 & 76.01 & 16.15 & $<0.0001$ \\
\hline Skull \#2 & 41 & 14.02 & 33.90 & 35.59 & 73.39 & 14.23 & $<0.0001$ \\
\hline Skull \#3 & 41 & 1.47 & 10.02 & 10.32 & 18.86 & 4.35 & $<0.0001$ \\
\hline Overall & 126 & 1.47 & 26.52 & 29.65 & 76.01 & 18.69 & $<0.0001$ \\
\hline
\end{tabular}

Table 2: Percent Weight Loss by Skull and Wilcoxon P-values.

\begin{tabular}{|l|l|l|l|l|l|l|l|}
\hline & N & Min. & Median & Mean & Max. & SD & P value \\
\hline Flow & 44 & 14.50 & 40.04 & 42.12 & 76.01 & 16.15 & $<0.001$ \\
\hline No Flow & 42 & 2.44 & 18.23 & 18.14 & 29.89 & 5.87 & \\
\hline Overall & 86 & 2.44 & 24.52 & 30.41 & 76.01 & 17.14 & \\
\hline
\end{tabular}

Table 3: Percent Weight Loss by Intensity and Wilcoxon P-values.

\begin{tabular}{|l|l|l|l|l|l|l|l|}
\hline & N & Min. & Median & Mean & Max. & SD & P value \\
\hline Flow & 44 & 14.50 & 40.04 & 42.12 & 76.01 & 16.15 & $<0.001$ \\
\hline No Flow & 42 & 2.44 & 18.23 & 18.14 & 29.89 & 5.87 & \\
\hline Overall & 86 & 2.44 & 24.52 & 30.41 & 76.01 & 17.14 & \\
\hline
\end{tabular}

Table 4: Percent Weight Loss by Flow Group and Student's t-test. radio density, expressed in this case by the amount of Hounsfield Units (HU) (mean $868 \mathrm{HU}$ vs. $850 \mathrm{HU}$ ). CT analysis of Skull \#3, revealed significantly greater bone thickness (mean $6.22 \mathrm{~mm}$ ) and higher radio density (mean 1208HU) compared to the others (Figure 5).

Similar to the differences in bone thickness and radio density, the acoustic measurements of the 3 samples showed higher values for focus intensity, peak negative and peak positive pressure for Skull \#1 and \#2 compared to Skull \#3. The acoustic data is shown in Table 1.

\section{Is HIFU sonothrombolysis depending on acoustic output power?}

To study the effect of acoustic output power on sonothrombolysis efficacy we decided to use Skull \#1 and a standard operating parameter setting (duty cycle $50 \%$, pulse width $200 \mathrm{~ms}$, duration $30 \mathrm{sec}$ ) with increasing acoustic output powers as follows: $100 \mathrm{~W}(\mathrm{~N}=46), 200 \mathrm{~W}$ $(\mathrm{N}=41), 235 \mathrm{~W}(\mathrm{~N}=40), 270 \mathrm{~W}(\mathrm{~N}=40)$, and 400W $(\mathrm{N}=41)$. Depending on the acoustic intensity at the focus the clot weight loss differed between $6.21 \%(100 \mathrm{~W})$ and $77.26 \%(400 \mathrm{~W})$ (Table 3). Adjusting for multiple comparisons using Holm's method all pairwise comparisons were found to be statistically significant. Higher intensities resulted in a larger weight percent loss.

\section{Is HIFU sonothrombolysis depending on flow mechanics?}

The experimental set up using a pulsatile flow pump producing a low velocity flow $(10 \mathrm{ml} / \mathrm{min})$ might occur in vivo, such as in case of partial recanalization or subtotal occlusion. The set up, however, does not mimick the event of flow stasis during acute thrombotic vessel occlusion. To produce flow stasis we simply turned off the flow pump during HIFU insonation. For this experiment we used Skull \#1 and tested for sonothrombolysis efficacy in two groups:

- Group 1 (N=44): AP 270W, DC 50\%, PW 200ms, ID 30sec, flow $10 \mathrm{ml} / \mathrm{min}$

- Group 2 (N=42): AP 270W, DC 50\%, PW 200ms, ID 30sec, no flow

Whereas a weight loss of $42.12 \%$ could be achieved in Group 1, the weight loss dropped to $18.14 \%$ in Group 2 during no flow conditions (Table 4).

\section{Discussion}

The results suggest that in vitro transcranial sonothrombolysis can be achieved using High Intensity Focused Ultrasound within seconds and without the use of a lytic agent, such as tPA. Sonothrombolysis efficacy depends on the amount of applied acoustic energy, variations in skull bone thickness and radio density as well as flow mechanics. Different from conventional diagnostic ultrasound systems transcranial defocusing and phase aberration seem to be negligible using the ExAblate $^{\mathrm{TM}} 4000$ HIFU headsystem.

\section{Does HIFU cause transcranial sonothrombolysis?}

In all 420 experiments a significant clot weight loss could be achieved. The thrombi inside the test tube were placed inside the hemispheric transducer in such a way that the center of the clot was exposed to the beam focus. During HIFU it could be monitored that clot lysis occurred exactly at the predicted location of the focus. In most of the cases, the visual defect caused by clot lysis described a gap of $4-5 \mathrm{~mm}$ which is equivalent to the diameter of the focus itself. This confirms that the acoustic energy drops dramatically and clot lysis does not occur outside the focus. Only in case of high acoustic output powers, such as $400 \mathrm{~W}$, the gap caused by the lytic defect enlarged. The 
mechanic impact on the clot itself, however, is rather vigorously using this high energy level.

\section{Does defocusing occur during transcranial HIFU?}

Clement et al described the effect of defocusing using hemispheric transducers in the sub MegaHertz frequency range first in 2002 [19]. To answer the question whether defocusing occurs during transcranial insonation using the ExAblate ${ }^{\mathrm{TM}} 4000$ HIFU headsystem, acoustic measurements were performed at focus before and after having an intervening skull in place. The fact that no considerable defocusing occured during transcranial HIFU is noteworthy and a major technical achievement. This might be mainly due to the geometry of the hemispheric transducer, providing access to the entire surface of the skull calvarium and, therefore, preferred angles of incidence. Another factor which contributes to this might be that the wave length of the ultrasound is greater than the skull thickness. Having a transmit frequency of $220 \mathrm{KHz}$ and assuming a speed of sound in human skull bone of about $3000 \mathrm{~m} / \mathrm{s}$ the actual wave length is about $13.6 \mathrm{~mm}$. The mean skull thicknesses measured for the three specimens used in this study, however, ranged between $5.49-6.22 \mathrm{~mm}$. Phase aberrations caused by non-uniform transit times through the skull may be expected to have less effect than the case of higher frequencies where wavelengths are considerable less than the skull thickness. How much higher transmit frequencies, such as for diagnostic ultrasound devices (ie. 2.0 MegaHertz), affect defocusing and phase aberration has been described extensively in the literature [14,20-23].

\section{Is HIFU sonothrombolysis efficacy depending on skull characteristics?}

Based on the results it can be assumed that sonothrombolysis efficacy is dependent on skull bone properties, such as thickness and/or density. Material properties of the human skull are not well understood and there seems to be no consensus across the literature. One attempt to better describe skull bone characteristics was suggested by Fry et al. [24], based on a three layer model of the skull (inner/outer table of cortical bone with trabecular bone sandwiched between). A relation between amount of clot lysis and skull bone thickness/density seemed to be obvious in the present study and was confirmed by the acoustic measurements showing lower acoustic intensities at the focus in case of increased skull thickness and radio density. Skull \#2 showed the lowest values for thickness and radio density, but did not reach the highest amount of clot lysis. This could be explained by suboptimal degassing of the specimen in preparation for the acoustic measurements. Air entrapment, for example in the trabecular bone layer, leads to total reflection of the ultrasound wave.

\section{Is HIFU sonothrombolysis depending on acoustic output power?}

The results confirm the impression that sonothrombolysis efficacy is related to the acoustic energy applied to the thrombus. Whether this relationship is linear is doubtful. It is rather to be expected that there might be a minimum as well as a maximum threshold. Regarding low energies, this threshold would simply be defined by a certain energy level below which no thrombolysis activity can be assessed, given that all other operating parameters remain constant. To define this minimum threshold was not an objective of this study. Similar to the minimum threshold, a maximum threshold might be defined beyond which no further clot lysis can be achieved. At higher energy levels temperature elevation might become an important factor. It is suggested by other investigators that thermal mechanisms, caused by ultrasound hyperthermia, contribute to enhanced thrombolysis [25-27]. Tissue heating, however, can be a safety issue in vivo during sonothrombolysis and has to be investigated carefully once this application will be translated into an animal model. This includes the assessment of potential endothelial or vessel wall damage as well as inflammatory, necrotic or apoptotic reactions of the exposed brain tissue. Potential blood-brain opening has to be investigated as well. Given the today's technology standard, the focus itself, although already small in diameter might not be restricted just to the clot itself but adjacent tissue structures as well. A weakness of the present study is that temperature changes during insonation and changing acoustic energies were not monitored. This is true as well for clot fragmentation which is another safety concern with regard to thrombolysis. The present study did not account for clot fragmentation.

\section{Is HIFU sonothrombolysis depending on flow mechanics?}

It could be demonstrated that flow mechanics have a significant impact on sonothrombolysis efficacy. In case of thrombotic vessel occlusion flow stasis can be expected. To mimick flow stasis the flow pump was turned off which resulted in a significant decrease of thrombolysis efficacy. Vice versa, thrombolysis increased significantly when flow was established. Although it might be rather intuitive that flow promotes thrombolysis, it is noteworthy that the impact of flow mechanics on thrombolysis has not been discussed in the literature until today. The impact of flow mechanics on sonothrombolysis should be a future research focus.

In summary, the results of this in vitro study suggest that transcranial sonothrombolysis can be achieved noninvasively using High Intensity Focused Ultrasound within seconds and without the use of tPA. Further investigations on HIFU sonothrombolysis should focus on the understanding of basic mechanisms, optimized operating parameter combinations and, most important, whether the knowledge gained in vitro can be translated into in vivo. The latter is of great interest especially from a safety point of view and with regard to potential tissue damage caused by transcranial HIFU sonothrombolysis.

\section{References}

1. Braaten JV, Goss RA, Francis CW (1997) Ultrasound reversibly disaggregates fibrin fibers. Thromb Haemost 78: 1063-1068.

2. Devcic-Kuhar B, Pfaffenberger S, Gherardini L, Mayer C, Gröschl M, etal. (2004) Ultrasound affects distribution of plasminogen and tissue-type plasminogen activator in whole blood clots in vitro. Thromb Haemost 92: 980-985.

3. Datta S, Ammi A.Y., Coussios C.C., Holland, C.K (2007) Monitoring and simulating stable cavitation during ultrasound-enhanced thrombolysis. Journal of the Acoustic Society of America 122 (3052).

4. Datta S, Coussios CC, McAdory LE, et al. (2006) Correlation of cavitation with ultrasound enhancement of thrombolysis. Ultrasound Med Biol 32: 1257-1267.

5. Prokop AF, Soltani A, Roy RA (2007) Cavitational mechanisms in ultrasoundaccelerated fibrinolysis. Ultrasound Med Biol 33: 924-33.

6. Eggers J, Koch B, Meyer K, Konig I, Seidel G (2003) Effect of ultrasound on thrombolysis of middle cerebral artery occlusion. Ann Neurol 53: 797-800.

7. Eggers J, Konig IR, Koch B, Handler G, Seidel G (2008) Sonothrombolysis with transcranial color-coded sonography and recombinant tissue-type plasminogen activator in acute middle cerebral artery main stem occlusion: results from a randomized study. Stroke 39: 1470-1475.

8. Alexandrov AV (2004) Ultrasound identification and lysis of clots. Stroke 35 2722-2725.

9. Alexandrov AV, Mikulik R, Ribo M, Sharma VK, Lao AY, et al. (2008) A pilot randomized clinical safety study of sonothrombolysis augmentation with ultrasound-activated perflutren-lipid microspheres for acute ischemic stroke. Stroke 39: 1464-1469. 
Citation: Hölscher T, Fisher DJ, Raman R, Ernstrom K, Zadicario E, et al. (2011) Noninvasive Transcranial Clot Lysis Using High Intensity Focused Ultrasound. J Neurol Neurophysiol S1. doi:10.4172/2155-9562.S1-002

10. Alexandrov AV, Molina CA, Grotta JC, Montaner J, Saqqur M, et al. (2004) Ultrasound-enhanced systemic thrombolysis for acute ischemic stroke. $\mathrm{N}$ Engl J Med 351: 2170-2178.

11. White DN (1967) The limitations of echo-encephalography. Ultrasonics 5: 8890.

12. Ballantine HT, Bolt RH, Hueter TF, Ludwig GD (1950) On the detection of intracranial pathology by ultrasound. Science 112: 525-528.

13. Ludwig GD, Bolt RH, Heuter TF, Ballantine HT (1950) Factors influencing the use of ultrasound as a diagnostic aid. Trans Am Neurol Assoc 51: 225-228.

14. White DN, Clark JM, White DA, Campbell JK, Bahuleyan K, et al. (1969) The deformation of the ultrasonic field in passage across the living and cadaver head. Med Biol Eng 7: 607-618.

15. Alexandrov AV (2002) Ultrasound-enhanced thrombolysis for stroke: clinical significance. Eur J Ultrasound 16: 131-140.

16. Alexandrov AV, Demchuk AM, Burgin WS, Robinson DJ, Grotta JC (2004) Ultrasound-enhanced thrombolysis for acute ischemic stroke: phase I. Findings of the CLOTBUST trial. J Neuroimaging 14: 113-117.

17. Eggers J, Seidel G, Koch B, Konig IR (2005) Sonothrombolysis in acute ischemic stroke for patients ineligible for rt-PA. Neurology 64: 1052-1054.

18. Daffertshofer M, Gass A, Ringleb P, Sitzer M, Sliwka U, et al. (2005) Transcranial low-frequency ultrasound-mediated thrombolysis in brain ischemia: increased risk of hemorrhage with combined ultrasound and tissue plasminogen activator: results of a phase II clinical trial. Stroke 36: 1441-1446.

19. Clement GT, Hynynen K (2002) A non-invasive method for focusing ultrasound through the human skull. Phys Med Biol 47: 1219-1236.

20. White DN, Curry GR, Stevenson RJ (1978) The acoustic characteristics of the skull. Ultrasound Med Biol 4: 225-252.

21. Holscher T, Wilkening WG, Molkenstruck S, Voit H, Koch C (2008) Transcranial sound field characterization. Ultrasound Med Biol 34: 973-980.

22. Clement GT, Hynynen K (2002) Correlation of ultrasound phase with physical skull properties. Ultrasound Med Biol 28: 617-624.

23. Clement GT, Sun J, Hynynen K (2001) The role of internal reflection in transskull phase distortion. Ultrasonics 3: 109-113.

24. Fry FJ, Barger JE (1978) Acoustical properties of the human skull. J Acoust Soc Am 63: 1576-1590.

25. Nahirnyak VM, Yoon SW, Holland CK (2006) Acousto-mechanical and thermal properties of clotted blood. J Acoust Soc Am 119: 3766-3772.

26. Nahirnyak V, Mast TD, Holland CK (2007) Ultrasound-induced thermal elevation in clotted blood and cranial bone. Ultrasound Med Biol 33: 1285-1295.

27. Sakharov DV, Rijken DC (2000) The effect of flow on lysis of plasma clots in a plasma environment. Thromb Haemost 83: 469-474. 